\title{
Salicylate-induced phantom auditory effects on reinforced behavior in pre- and postweanling rats
}

\author{
JAMES F. BRENNAN and JOHN J. BYRNES \\ University of Massachusetts, Boston, Massachusetts \\ and \\ PAWEL J. JASTREBOFF \\ University of Maryland School of Medicine, Baltimore, Maryland
}

\begin{abstract}
In two behavioral experiments, normal hearing or salicylate-induced phantom auditory perception were investigated in young pigmented rats. Twenty-four hours prior to training in Experiment 1, 45 preweanling pups (14-21 days of age) were exposed to continuous background noise. They were injected with sodium salicylate or saline either before or after training to suppress approach responses to a lactating dam during noise offset periods. The sequence of injection initiation affected acquisition and extinction rates, and the oldest subjects behaved similarly to adults reported in studies of salicylate-induced auditory effects. Adapting the task in Experiment 2 for 18 postweanling pups 32 days of age produced greater suppression and prolonged extinction when salicylate was administered prior to both acquisition and extinction sessions, whereas subjects injected with saline before training and salicylate before extinction showed rapid recovery from suppression. The experiments support the notion that the auditory system undergoes functionally important change at around 15-16 days of age.
\end{abstract}

We have reported behavioral evidence from adult pigmented rats that phantom auditory sensations induced by salicylate injection can influence the behavior of rats trained to associate periods of silence with aversive events, in a manner similar to that predicted if the animals were perceiving a sound (Jastreboff, Brennan, Coleman, \& Sasaki, 1988). In the adult paradigm, sodium salicylate was injected at dose levels suggested by studies that delineated its uptake in blood serum, cerebrospinal fluid, and the perilymph, under a variety of metabolic states and during various behavioral tasks (Jastreboff, Issing, Brennan, \& Sasaki, 1988). Our initial concern in this research was to establish an animal model of phantom auditory perception, or tinnitus, which is reported in humans as sound perceived in the absence of any external auditory stimulus. Our goal was to use the animal model of tinnitus to find its mechanisms and eventually test intrusive clinical interventions. The research led to a number of intriguing issues, not the least of which is the developmental context of this question of phantom auditory perception.

\footnotetext{
This study was supported by Grant DC00299 from the National Institute on Deafness and Other Communication Disorders. Preliminary work supporting Experiment 1 of the present study formed part of a thesis by J.J.B. submitted in fulfillment of requirements for the Honors Program in Psychology of the University of Massachusetts at Boston. The authors wish to thank Alex Mansfield for his assistance in conducting Experiment 2. P.J.J. is in the Department of Surgery at the University of Maryland School of Medicine. Correspondence should be addressed to J. F. Brennan, Department of Psychology, 100 Morrisey Boulevard, University of Massachusetts, Boston, MA 02125.
}

The basic experimental demonstration of the behavioral measure of phantom auditory sensation in adults used a conditioned suppression paradigm to assess salicylateinduced effects that depended on the time of initial onset of the subjective auditory effect. Subjects were trained to suppress ongoing lick responses through the pairing of a conditioned stimulus (CS) consisting of the offset of background noise and a pulse of footshock. When daily salicylate injections of the adult rats began before suppression training, the salicylate-induced auditory effect produced greater suppression of drinking behavior and slower recovery of licking during successive daily extinction sessions. Conversely, when injections began after suppression training, but before extinction testing, the salicylate-induced effect seemed to provide a cue about the changed experimental contingencies, resulting in less resistance to extinction and quicker recovery of drinking, relative to controls injected with the saline vehicle only. The phantom auditory character of the salicylate effect is inferred from the interference in the detection of the noise offset periods during extinction testing. We have since completed a fairly extensive study in which dose levels were systematically related to the extent of the salicylate-induced effect (Jastreboff \& Brennan, 1992); the dose threshold level producing reliable behavioral effects in adults was between 100 and $150 \mathrm{mg} / \mathrm{kg}$. Moreover, we have replicated the basic findings with quinine, also known to have auditory effects in humans (Jastreboff, Brennan, \& Sasaki, 1991). With both salicylate and quinine, the drug-induced effects were resistant to changes 
in motivation and variation in the auditory background of subjects. The effect was absent when subjects were trained with visual stimuli, supporting the salicylateinduced phenomenon as specifically auditory (Jastreboff \& Brennan, 1992; Jastreboff, Brennan, et al., 1988).

The overall purpose of the present experiments was to compare factors in the acquisition and retention of auditory mediated behavior in young rats. To do so, we proposed to extend the behavioral paradigm of phantom auditory perception in adult rats to preweanling and postweanling pups at age levels when we believe that both the auditory system and motor behavior are becoming functional. By 14 days of age, rat pups' eyes are beginning to open (Foreman \& Altaha, 1991), and other sensory systems begin to acquire behavioral utility as well.

\section{EXPERIMENT 1 \\ Salicylate-Induced Behavioral Effects on Acquisition and Extinction of Suppression in Preweanling Pups}

In transposing the essential features of the adult paradigm to accommodate preweanling rats, we included procedures that paralleled the distinction that resulted in different salicylate-induced effects in adults that depended on the time when the drug was introduced relative to behavioral training. Adapting an approach-avoidance task for preweanling pups (Collier, Mast, Meyer, \& Jacobs, 1979), we established an approach response by permitting young rats access to an anesthetized dam after they had traversed an inclined distance. There is evidence (e.g., Phifer, Denzinger, \& Hall, 1991) that rats as young as 6 days of age are able to direct feeding behaviors when mildly deprived of food and maternal stimulation. The approach tendency was then suppressed by associating a mildly aversive footshock with the offset of the background noise. We then tested for extinction of the response to noise offset. While cognizant that comparisons of behavioral differences at age levels of rapid growth present the issue of differential incentive motivation in the significance of results from rats ranging in age between 14 and 21 days, we believed that the behavioral measures employed would nevertheless support interpretations of meaningful developmental change.

\section{Method}

Subjects. A total of 45 male pigmented preweanling rat pups ranged in age from 14 to 20 days on Day 1 of the experiment. The subjects were born in the animal colony of the University of Massachusetts at Boston and were progeny of Charles River strains. For the duration of the gestation period and throughout the experiment, each litter and dam were housed in individual, acrylic maternity cages with dimensions of $15 \times 22 \times 44 \mathrm{~cm}$. The floor bedding was changed once each during the gestation, preweaning (Day 2 postnatally), and weaning (Day 25 postnatally) periods. The litters were sexed and culled to no more than 8 males on Day 2 postnatally. Beginning $24 \mathrm{~h}$ before training and for the remainder of the experiment, the subjects were exposed to continuous background noise of $60 \mathrm{~dB}$ SPL. For all subjects and dams, food and water were available ad lib throughout the experiment, with the exception of the duration beginning $2 \mathrm{~h}$ prior to training and testing when the pups were separated from their dams. The subjects were maintained on a 12:12-h light:dark cycle (lights on at 7:00 a.m.) throughout the experiment. At the conclusion of extinction testing, approximately $2.5 \mathrm{~h}$ after injections, the subjects were anesthetized with an overdose of nembutal, blood samples were taken from the tail tip. and serum was separated for analysis of salicylate levels. The results of the analysis indicated fairly consistent levels across the age categories: 15 days, $M=40.9 \mathrm{mg} / \mathrm{dl}, S E M=1.62 ; 18$ days. $M=39.7 \mathrm{mg} / \mathrm{dl}, S E M=2.22 ; 21$ days, $M=41.9 \mathrm{mg} / \mathrm{dl}, S E M=$ 1.4. These levels were in agreement with expectations based on peak salicylate levels obtained from adult rats 2-3 $\mathrm{h}$ after injection (Jastreboff, Issing, et al., 1988) and within the range observed in humans treated with salicylate (Mongan, Kelly, Nies, Porter, \& Paulus, 1973).

Apparatus. Acquisition training and extinction testing took place in a $60 \times 30 \times 30 \mathrm{~cm}$ glass chamber containing a $30 \times 35 \mathrm{~cm}$ ramp placed at a $15^{\circ}$ incline. The ramp was covered with a rubber corrugated mat that provided a textured surface to help the youngest subjects, especially, to move up the incline. At the top of the ramp, a $15 \times 30 \mathrm{~cm}$ platform consisting of steel rods spaced $1 \mathrm{~cm}$ apart delivered the scrambled footshock, unconditioned stimulus (US) of $1.0 \mathrm{~mA}$ from a BRS/LVE Generator (Model SBS-004). This level of shock and the durations observed in this study are within the range reported for rat pups of this age level (Miller \& Spear, 1989; Myslivecek \& Hassmannová, 1990; Richardson, Siegel, \& Campbell, 1989; Rocha \& Vendite, 1990) in various tasks, as well as for approach-avoidance training (Weihmuller \& Collier, 1990). Immediately beyond the grid surface, a Plexiglas platform supported a heating pad on which an anesthetized (i.p. Nembutal, $50 \mathrm{mg} / \mathrm{kg}$ ) dam reclined. The experiment was conducted in an isolated room that attenuated extraneous sounds, and the only illumination within the room was provided by a $25-\mathrm{W}$ houselight aimed away from the apparatus but sufficiently bright to permit videotaping of each acquisition and extinction trial with a Sharp Camcorder (Model VL 170U). Background noise presentations in the experimental chamber and in the home litter cage prior to training were provided by Hewlett-Packard precision noise generators (Model 8057A), which delivered noise to two 9-cm-wide Motorola speakers (P35-VAH). One speaker was mounted $75 \mathrm{~cm}$ above the center of the glass chamber (i.e., $45 \mathrm{~cm}$ from the top of the walls) and directed downward to the chamber. Another speaker was centered $75 \mathrm{~cm}$ above the litter cage housing the pups $24 \mathrm{~h}$ prior to the acquisition and extinction sessions. The sound pressure levels close to the top of the center of the chamber and the litter cage were measured by a precision sound level meter (Quest Electronics, Model 155) in 1/3 octave bands (Quest Electronics 1/3 octave filter, Model OB-133). The SPL level was calculated for frequencies of $1-16 \mathrm{kHz}$, according to standard procedures (Durrant \& Lovrinic, 1984), and it was maintained at $60 \mathrm{~dB}$ SPL. A light-emitting diode measuring $1.0 \mathrm{~mm}$ in diameter was placed over the experimental chamber as an indicator of CS/US onset. This light was not visible to subjects, but provided a cue for the onset of shock when the videotape was subsequently reviewed.

Procedure. Twenty-four hours prior to training, all subjects in the litter were exposed to continuous noise of $60 \mathrm{~dB}$ SPL generated through the $9-\mathrm{cm}$ speaker suspended above the cage. The experimental design involved the use of three age levels of preweanling pups exposed to one of three salicylate administrations ( $n=5 /$ group). The subjects were 14, 17, or 20 days of age on acquisition sessions. Two hours prior to both the acquisition and the extinction sessions, 5 pups at each age level were injected subcutaneously (s.c.) with $233-\mathrm{mg} / \mathrm{kg}$ sodium salicylate, which corresponds to $200 \mathrm{mg} / \mathrm{kg}$ of salicylate acid. These subjects are henceforth designated the salicylate before training (SB) treatment. Groups of 5 additional subjects at each age level received injections of the saline vehicle prior to the acquisition session, but $233-\mathrm{mg} / \mathrm{kg}$ injections of salicylate prior to the extinction session on the second day of behavioral data collection and will 
be referred to as the salicylate after training (SA) treatment. The third, the control condition, involved s.c. saline injections prior to both sessions. It should be noted, then, that the SA subjects were treated just like the controls on Day 1 of the experiment, but like the SB subjects on Day 2 of the experiment.

All subjects were trained and tested individually. Training involved two stages, both done on Day 1 of the experiment. Two hours prior to the training session, a subject received the appropriate injection and was placed in an incubator next to the litter cage for the 2-h deprivation period away from its dam and litter mates. At the beginning of training, each subject was placed at the base of the ramp and allowed unlimited time to explore and eventually find the dam, which took no longer than $5 \mathrm{~min}$ for all subjects. Acquisition of the approach response to the dam was defined as 2 successive trials on which the subjects proceeded directly up the ramp, crossed the grid, and accessed the dam in less than $30 \mathrm{sec}$ without any marked pauses in their progression. A pause was noted when subjects halted in their forward movement for longer than $1 \mathrm{sec}$. The subjects were allowed $10 \mathrm{sec}$ with the dam as reinforcement. Suppression training involved the nearly simultaneous offset of the background noise (CS) when the subjects stepped onto the grid, which was activated with the footshock US. When the subjects reached the top of the ramp, just prior to stepping onto the grid, the background noise was turned off. If the subject retreated down the incline, the noise was reactivated; if it proceeded on the grid, activated with shock, the noise remained off. If the subjects remained on the grid for $10 \mathrm{sec}$ because of disorientation, the trial was automatically terminated. Suppression training trials continued until the subjects paused at the top of the ramp just before the grid for at least $4 \mathrm{sec}$ on 4 consecutive trials. Subjects that did not meet the acquisition criterion within 25 trials were discarded from the experiment and replaced with naive pups; 3 subjects were discarded for this reason.

The second experimental session on Day 2 involved extinction of the suppression and recovery of the approach response. Two hours prior to the session, a subject was removed from the litter cage, and the animals assigned to the SB and SA treatments were injected with $233 \mathrm{mg} / \mathrm{kg}$ of salicylate, while the control subjects received a second saline injection. The extinction procedure was the same as the acquisition training, except that the shock was turned off. Extinction trials continued until 4 consecutive trials were recorded without any apparent pause longer than $1 \mathrm{sec}$ at the top of the ramp.

Each trial was videotaped, and two experimenters reviewed the tapes separately to record data on the pause responses. The dependent measures collected in this experiment included trials to criterion, latency measures on trials, and pause durations for both sessions.

\section{Results and Discussion}

Session 1: Acquisition. All subjects were able to navigate the ramp and access the dam within two trials following the initial exploratory trial. Table 1 summarizes the mean number of trials, with related $S E M$ s, to acquire suppression of the approach response in each group. While both the fastest (SA) and slowest (SB) groups were at the youngest age level, the analysis of these data revealed only a significant drug effect $[F(2,36)=3.97, p<.03]$, which may be attributed to higher numbers of trials in the SB groups overall than in the others (Duncan tests, all $p s<.05)$. For this session, none of the saline-injected groups (SA and control treatments) differed from each other. Neither the main effect of age $(F<1)$ nor the drug $\times$ age interaction attained significance.

To accommodate the different rates of acquisition trials, in the examination of trial latencies, the total number of trials for each animal was blocked into equivalent quarters of trials, and mean trial latencies per quarter of trials were calculated. Consistent with the trials-to-acquisition data, the analysis of the mean latencies of successive quarters of trials revealed a significant drug effect $[F(2,36)=$ $10.25, p<.0003$ ], as well as the expected effect of progressive quarters of training $[F(3,108)=11.56, p<$ $.0001]$. Duncan tests confirmed that these effects were due to significantly longer latencies overall in the salicylate (SB) groups than in the groups injected with saline only (SA and control treatments), and latencies in the last three blocks were longer than in the first block for all groups $(p s<.05)$, reflecting the acquisition process. Neither the main effect of age nor any of the interactions attained significance.

The respective means and $S E M$ s for the pause duration before the shock grid area at each age level were: 14 days, $4.48 \mathrm{sec}, 0.79 ; 17$ days, $2.89 \mathrm{sec}, 0.57 ; 20$ days, $3.10 \mathrm{sec}, 0.52$. The mean pause durations during successive quarters of acquisition trials were analyzed in the same manner as were the mean trial latencies during successive quarters. Analysis of these data indicated significant effects of age $[F(2,36)=10.25, p<.0003]$ and of successive quarters $[F(3,108)=11.56, p<.0001]$. Duncan tests confirmed that this effect was due to longer pauses in the 14-day groups on the first three blocks (all $p s<.05)$. Neither the main effect of drug nor any of the interactions attained significance.

As part of the family of frequently used drugs, including acetylsalicylate acid, salicylates are generally prescribed for humans because of their anti-inflammatory and painreducing actions (Flower, Moncada, \& Vane, 1980; Vane, 1971). If present in this experiment, the analgesic properties of salicylate could have been reflected in differences in US duration, which could last as long as $10 \mathrm{sec}$. An

Table 1

Mean Numbers of Acquisition Trials and

Related SEMs in Experiment 1 for Groups $(n=5)$ at

Each Age Level and Drug Treatment With Salicylate

Injected Before Both Sessions (SB), Before the Extinction Test

Only (SA), or Saline-Injected Before Both Sessions (Controls)

\begin{tabular}{|c|c|c|c|c|c|c|}
\hline \multirow[b]{2}{*}{ Days of Age } & \multicolumn{2}{|c|}{ SB } & \multicolumn{2}{|c|}{ SA } & \multicolumn{2}{|c|}{ Controls } \\
\hline & $M$ & SEM & $M$ & $S E M$ & $M$ & $S E M$ \\
\hline 14 & 20.00 & 1.81 & 9.20 & 2.80 & 10.20 & 2.73 \\
\hline 17 & 11.20 & 1.53 & 9.80 & 2.64 & 11.40 & 1.81 \\
\hline 20 & 13.40 & 1.81 & 11.40 & 2.50 & 11.80 & 2.22 \\
\hline
\end{tabular}


analysis of the mean duration of footshock in quarters of acquisition trials indicated significant effects of age $[F(2,36)=10.43, p<.0003]$ and drug $[F(2,36)=$ $26.80, p<.0001]$, as well as a significant interaction between them $[F(4,36)=8.77, p<.0001]$. An effect of successive blocks of acquisition trials $[F(3,108)=$ $24.11, p<.0001]$ was also found, as were the interactions of blocks $\times$ drug $[F(6,108)=2.11, p=.058]$ and blocks $\times$ age $\times$ drugs $[F(12,108)=2.86, p<$ $.0029]$. Figure 1 shows the mean times for the duration of the shock at each of the four blocks of acquisition trials for the youngest (bottom panel), 17-day (middle panel), and oldest (top panel) age levels within the respective drug treatments of SB (filled squares), SA (open triangles), and saline-injected controls (open circles). Note that both the control and the SA groups received saline injections prior to the acquisition training day. Duncan tests indicated that the youngest group had longer shock durations at Blocks 1,3 , and 4 (all $p s<.05$ ), suggesting perhaps a weaker association between the CS and US in the SB group. Furthermore, all SB groups had longer durations at each block of training trials (all ps $<.05$ ) than did the SA and control groups, which, in turn, did not differ from each other. As supported in Figure 1, while the 14-day SB group had

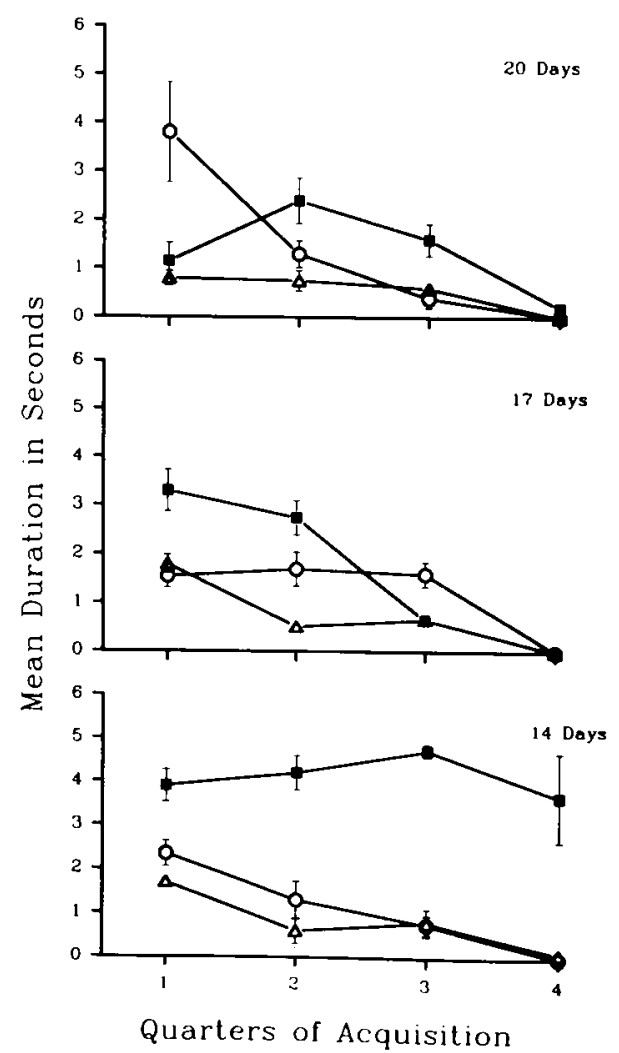

Figure 1. Mean duration and associated SEMs for US action in Experiment 1 for groups injected with salicylate (SB group, filled squares) or with saline (SA group, open triangles; saline-injected controls, open circles) at each age level, 14 (bottom panel), 17 (middle), and 21 days of age (top), during successive quarters of acquisition. the longest shock duration across blocks and a mean value of $3.65 \mathrm{sec}$ on Block 4 , the mean shock duration for the $\mathrm{SA}$ and control groups by Block 4 was $0.039 \mathrm{sec}$, which includes the four nonshock criterion trials. Given the relatively little exposure to footshock overall, and the pattern of more shock exposure in the SB treatments at all age levels, it is possible that in the youngest SB group nonspecific factors may have contributed to the shock durations. That is, vestibular problems or analgesia, if present, are more dependent on salicylate than on other possibilities, such as acclimation to successive aversive treatments (cf. Maier \& Watkins, 1991).

A second concern in the interpretation of acquisition in the youngest group is derived from evidence of less developed, prepotent fleeing responses in 15-day-old rats in comparison with older pups tested in this type of approach-avoidance conflict task (Weihmuller \& Collier, 1990). This possibility was not supported by the present acquisition results, since the nonsalicylate-injected groups (SA and controls) were not different from their older counterparts in terms of trial latencies or the length of US duration (Figure 1). Nor does this possible explanation help one understand the longer times of US exposure across blocks of trials in the youngest SB group.

Thus, while all subjects, regardless of age, acquired the conditioned suppression signaled by a CS complex including noise offset, the analyses of acquisition data suggest disruptive effects from salicylate administered before training. Interestingly, the duration of footshock in acquisition was longest in the youngest subjects, possibly owing to the strongest extent of nonspecific drug effects. A more subtle effect of salicylate on US duration did emerge in Blocks 1 and 2 for the 17-day group and in Blocks 2 and 3 for the oldest subjects, which may reflect an associative auditory character.

Session 2: Extinction. All subjects met the extinction criterion defined as four consecutive trials with pause latencies at the top of the ramp of no longer than $1 \mathrm{sec}$. Analysis of the numbers of trials to the extinction criterion, seen in Table 2, did not indicate any significant differences from either of the independent variables.

For the analysis of the trial latency data, blocked into four successive quarters of extinction trials, significant effects of age $[F(2,36)=4.07, p<.025]$ and drug $[F(2,36)=3.13, p<.06]$ were found, but not their interaction. The effect of successive quarters of trials was expected $[F(3,108)=4.81, p<.003]$ and a blocks $\times$ age effect $[F(6,108)=3.01, p<.01]$ also emerged. There was also a significant blocks $\times$ age $\times$ drug interaction $[F(12,108)=2.36, p<.009]$, which is depicted in Figure 2. Figure 2 suggests that the expected trend, based on adult results (Jastreboff, Brennan, et al., 1988), was found to some extent at the two older age levels in the present experiment-namely, salicylate tends to produce greater resistance to extinction of suppression if the salicylate-induced effect is present during both acquisition and extinction of the suppression (SB groups). When salicylate is injected prior to the extinction session only 
Table 2

Mean Numbers of Extinction Trials and Related SEMs for Groups Under Each Combination of Age Level and Salicylate Treatment in Experiment 1

\begin{tabular}{|c|c|c|c|c|c|c|}
\hline \multirow[b]{2}{*}{ Days of Age } & \multicolumn{2}{|c|}{ SB } & \multicolumn{2}{|c|}{ SA } & \multicolumn{2}{|c|}{ Controls } \\
\hline & $M$ & SEM & $M$ & $S E M$ & $M$ & $S E M$ \\
\hline 15 & 5.00 & 0.31 & 5.00 & 0.44 & 6.80 & 1.32 \\
\hline 18 & 6.60 & 0.92 & 8.80 & 2.56 & 7.80 & 1.24 \\
\hline 21 & 6.20 & 0.86 & 7.00 & 0.83 & 8.25 & 1.71 \\
\hline
\end{tabular}

Note-SB, salicylate injected before both sessions; SA, salicylate injected before the extinction test only; controls, saline injected before both sessions.

(SA groups), the effect is almost opposite, in that extinction is facilitated.

The duration of the pause before the grid area during extinction supported the overall trial latency results. Although the main effects of age and drug, or their interaction, failed to attain significance, the effect of successive blocks of mean pause latencies was significant $[F(3,108)=12.69, p<.0001]$, as was the blocks $\times$ age $\times$ drug interaction $[F(12,108)=2.19, p<.016]$. Again for this measure, the maximum difference between longest latencies in the SB treatment and shortest in the SA treatment was clearest in the oldest groups. Given that the experimental contingencies had not changed for the

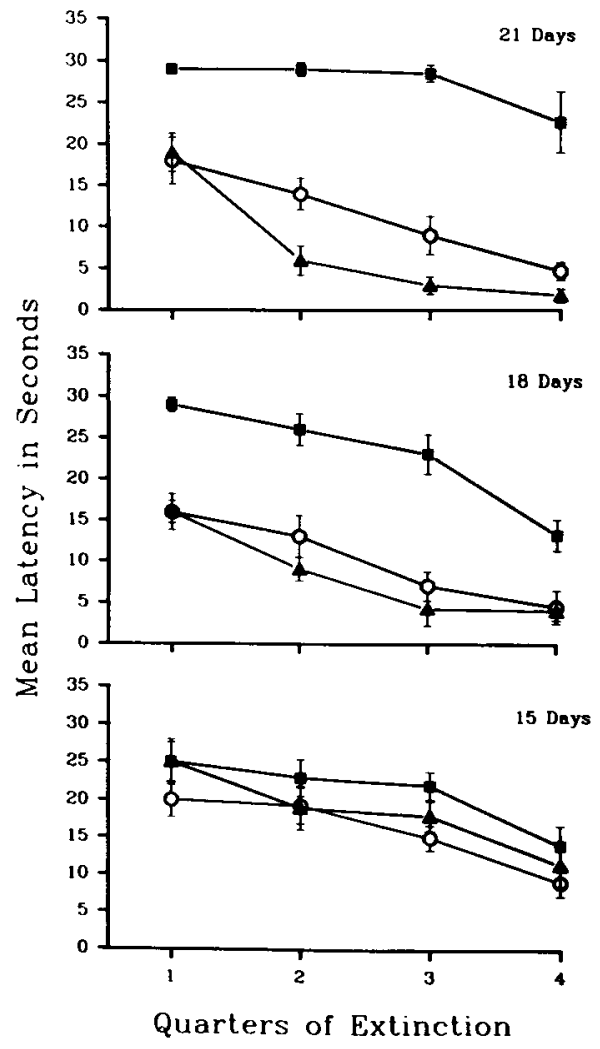

Figure 2. Mean total trial latencies and SEMs across quarter blocks of extinction trials for the two salicylate-injected groups (SB, filled squares; SA, filled triangles), and the saline-injected control group (open circles) at each age level of Experiment 1.
SB or control groups (i.e., the quality of injection prior to extinction was identical to that given before acquisition), it may be inferred that the novel auditory cue, presumably induced by salicylate, resulted in diminished pause latencies by the second quarter of extinction testing in the SA group.

One final point in Experiment 1 concerns the possibility of cumulative effects from multiple injections of salicylate. In adult rats, there is no evident of long-term cumulative salicylate effects (Jastreboff, Hansen, Sasaki, \& Sasaki, 1986; Jastreboff \& Sasaki, 1986). Salicylate uptake in serum, cerebrospinal fluid, and perilymph reaches a maximum level in conscious, active adult rats about $2 \mathrm{~h}$ after injection, and salicylate levels tend to have a half life of about $6 \mathrm{~h}$ (Jastreboff, Issing, et al., 1988). Although we did not suspect that multiple injection effects would be different in preweanling pups, we nevertheless conducted an examination to determine whether chronic salicylate injections might have a cumulative effect on the behavior of young rats. Briefly, four groups of 5 pups began injections of salicylate $(233 \mathrm{mg} / \mathrm{kg})$ or saline at 14 days of age and continued until 21 days of age. Three groups started salicylate injections at 14,17 , or 20 days of age, and the fourth group received saline injections throughout the 7-day series. Training and testing, exactly as described for Experiment 1, proceeded for all groups on Days 20 and 21, respectively. The salicylate effects that were found in this examination agreed with expectations based on findings at the oldest age level obtained in Experiment 1-namely, the results of the salicylate groups appeared similar to the results of the oldest SB group, while the group with 7 days of saline injections had acquisition and extinction functions predicted from the control group of Experiment 1. Thus, our evaluation confirmed that salicylate injections have little long-term effect within the preweanling age range examined.

\section{EXPERIMENT 2 \\ Salicylate-Induced Behavioral Effects on Acquisition and Extinction of Suppression in Postweanling Pups}

The general findings of Experiment 1 indicate that functional associative value from salicylate-induced effects in preweanling pups seemed to emerge after $\mathbf{1 5}$ days of age and clearly conformed to expectations based on adult 
trends by the ages 20-21 days. In Experiment 2, we attempted to examine salicylate effects in postweanling pups through procedures designed to parallel those with preweanlings in Experiment 1.

\section{Method \\ Subjects. A total of 18 male pigmented postweanling rat pups were 32 days of age on the 1st day of the experiment. The subjects were borm in the animal colony of the University of Massachusetts at Boston from parents descended from Charles River strains. The litters were sexed and culled to no more than 8 males on Day 2 post- natally, at which time the cage bedding was changed. The subjects were weaned at Day 23 and housed in metal gang cages in groups of 4 litter mates until the day before behavioral training, when they were transferred to a litter cage. At the start of experimentation, the subjects' weights ranged from 73 to $85 \mathrm{~g}(M=81.8 \mathrm{~g}, S E M=2.3)$. Except for specific periods of water deprivation, the subjects had food and water available ad lib, and all subjects were maintained on a 12:12-h light:dark cycle (lights on at 7:00 a.m.) throughout the experiment.}

Apparatus. Acquisition training and extinction testing were conducted in a straight alley maze, $92 \mathrm{~cm}$ long $\times 16 \mathrm{~cm}$ wide $\times 15 \mathrm{~cm}$ high. The walls and ceiling were made of clear acrylic, and the entire floor was composed of $2-\mathrm{mm}$ steel rods spaced $1 \mathrm{~cm}$ apart. A 14-cm-long start compartment was separated from the rest of the maze by a clear acrylic guillotine door, and the rods in the last $18 \mathrm{~cm}$ of the maze were wired to deliver a 1.0-mA scrambled footshock from a BRS/LVE Generator (Model SBS-004). The entire maze was suspended over a tray covered with hardwood bedding (AB-SORBDRI). On the end wall, beyond the wired portion of the grid floor, a small hole $4 \mathrm{~cm}$ above the floor was drilled, through which a stainless steel drinking tube of the same variety as was used in the home cage was inserted. The tube was connected to a bottle suspended outside the maze. The ceiling was composed of a total of five hinged lids to provide access to a subject at any point in the maze. On two of the lids, 9-cm-wide Motorola speakers (P35-VAH) were mounted over perforated holes drilled though the plastic. Each speaker was centered $25 \mathrm{~cm}$ from each end of the maze. The experiment was conducted in an isolated room that attenuated extraneous sounds, and the only illumination within the room was provided by a $25-\mathrm{W}$ houselight aimed away from the apparatus but sufficient to permit videotaping of each acquisition and extinction trial by a Sharp Camcorder (Model VL-170U). The remaining equipment used to present auditory stimuli was the same as that used in Experiment 1. Background noise presentations in the experimental chamber and in an isolation cage prior to training were provided by Hewlett-Packard precision noise generators (Model 8057A), which delivered noise to another 9-cm-wide Motorola speaker (P35-VAH) centered $75 \mathrm{~cm}$ above the holding cage housing the pups $24 \mathrm{~h}$ prior to the acquisition session. The sound pressure levels measured on the floor at the center of the maze and in the holding cage were maintained at $60 \mathrm{~dB}$ SPL evaluated at frequencies of $1-16 \mathrm{kHz}$. A light-emitting diode measuring $1.0 \mathrm{~mm}$ in diameter was placed over the experimental chamber as an indicator of US onset. This light was not visible to the subjects; it provided a cue for the onset of shock when the videotape was subsequently reviewed.

Procedure. Each subject was trained and tested individually, but groups of 4 pups were run per 2-day sessions. Experiment 2 followed a conditioned suppression training procedure that was analogous to that used in Experiment 1. Approximately $24 \mathrm{~h}$ prior to a training session, 4 subjects were removed from the gang cage, weighed, deprived of water, and housed together in a litter cage while exposed to continuous noise of $60 \mathrm{~dB}$ SPL generated through the 9-cm speaker suspended above the cage. The subjects were assigned in successive order to one of three salicylate administration treatments ( $n=6 /$ group). Two hours prior to both the acquisition and the extinction sessions, 6 pups were weighed and then injected s.c. with $233-\mathrm{mg} / \mathrm{kg}$ salicylate (SB group). Another group of 6 subjects received injections of the saline vehicle prior to the acquisition session, but $233-\mathrm{mg} / \mathrm{kg}$ salicylate prior to the extinction session on the 2 nd day of behavioral data collection (SA group). The third, the control group, was weighed and then given saline injections prior to both sessions. Thus, as in Experiment 1, the SA subjects were treated like the controls on Day 1 of the experiment, but like the SB subjects on Day 2 of the experiment.

Two hours prior to the training session, the subjects received the appropriate injection and kept singly in a separate litter cage. At the beginning of training, each subject was placed in the start compartment of the maze with the background noise on continuously and allowed up to $5 \mathrm{~min}$ to explore and eventually find the drinking tube containing a sweetened water solution. Acquisition of the approach response to the drinking tube was defined as 2 successive trials on which the subjects proceeded directly to the drinking tube upon opening of the guillotine door. The subjects were allowed 4-5 sec of drinking the liquid reinforcement. Suppression training involved the simultaneous offset of the background noise when subjects stepped onto the grid, which was activated with the 1.0-mA scrambled shock. If the subject retreated from the grid area back toward the start compartment, the noise was reactivated; if it proceeded on the grid with the shock on, the noise remained off. If the subjects remained on the grid for $10 \mathrm{sec}$ because of disorientation, the trial was automatically terminated. Suppression training continued until the subjects paused before the active grid area for at least $4 \mathrm{sec}$ on two consecutive trials. Subjects that did not meet the acquisition criterion within 25 trials were discarded from the experiment and replaced with naive pups; only 1 subject was discarded for this reason. Following the acquisition training session, the subjects were allowed $30 \mathrm{~min}$ of access to plain tap water in the holding cage.

On the 2nd day of the experiment, extinction of the suppression and recovery of the approach response were tested. Two hours prior to the session, each subject was removed from the litter cage and weighed, and the SB and SA subjects were injected with $233 \mathrm{mg} / \mathrm{kg}$ of salicylate, while the controls received a second saline injection. The extinction trials involved the same sequence of events as did the acquisition trials, but the shock was disconnected. Each session continued until four consecutive trials were recorded without any apparent pause of more than $1 \mathrm{sec}$ at the end of the alley. All trials were videotaped, and two experimenters reviewed tapes separately to record data on the pause responses. The same dependent measures as in Experiment 1 were collected in this experiment, including trials to criterion, latency measures on trials, and pause durations for both sessions.

\section{Results and Discussion}

Subjects easily acquired the approach response. The numbers of trials to the acquisition and extinction criteria for the conditioned suppression response are shown along with the respective SEMs in Table 3. Analyses of these data did not indicate any significant trends. Moreover, acquisition measures of the number and durations of footshock exposure did not reveal any significant effects.

As with previous experiments, trial latencies were blocked into units of trials, so that mean latencies per block would reflect each subject's latency at a given portion of acquisition or extinction, despite different overall rates of learning. For this experiment, total trials were blocked into units of one sixths of acquisition or of extinction. Analysis of the blocked sixths of acquisition trial 
Table 3

Mean Numbers of Acquisition Trials and Related SEMs During the Acquisition and Extinction Sessions for Each Drug Treatment Group of Experiment 2

\begin{tabular}{|c|c|c|c|c|c|c|}
\hline \multirow[b]{2}{*}{ Session } & \multicolumn{2}{|c|}{ SB } & \multicolumn{2}{|c|}{ SA } & \multicolumn{2}{|c|}{ Controls } \\
\hline & $M$ & $S E M$ & $M$ & $S E M$ & $M$ & $S E M$ \\
\hline $\begin{array}{l}\text { Acquistion } \\
\text { Extinction }\end{array}$ & $\begin{array}{l}13.8 \\
12.63\end{array}$ & $\begin{array}{l}1.33 \\
2.71\end{array}$ & $\begin{array}{r}12.53 \\
9.77\end{array}$ & $\begin{array}{l}2.41 \\
2.03\end{array}$ & $\begin{array}{l}11.0 \\
17.4\end{array}$ & $\begin{array}{l}1.18 \\
1.73\end{array}$ \\
\hline
\end{tabular}

Note-SB, salicylate injected before acquisition training; SA, salicylate injected after acquisition training; controls, saline injected before both acquition and extinction.

latencies revealed only the effect of progressive increases in trial latencies as subjects acquired the suppression response $[F(5,75)=14.2, p<.0001]$. The mean latencies of the pause before the shock-activated area of the grid floor proved more sensitive during acquisition. The main effects of drug treatment $[F(2,15)=6.8, p<.008]$ and successive blocks of training $[F(5,75)=48.1, p<$ $.0001]$ were significant, as was the interaction between these variables $[F(10,75)=2.07, p<.04]$. Figure 3 shows the mean pause latencies for each sixth of acquisition and extinction trials among the groups having only the saline vehicle (controls, open circles; SA, open triangles) or the salicylate (SB, filled squares) injected $2 \mathrm{~h}$ before acquisition training. The extinction symbols for the right panel of Figure 3 are the same, except that, as in Experiment 1, the SA triangles are filled because that group received salicylate $2 \mathrm{~h}$ prior to the extinction session. As Figure 3 indicates, the trial latencies on the second block of acquisition trials were largely responsible for the interaction effect, which was confirmed by the Duncan tests showing that at this stage of training, the SB group had longer latencies (all $p s<.05$ ) than did the other two groups, which did not differ. The mean pause latencies during noise offset over six blocks of extinction trials (Figure 3 ) indicated a consistent pattern of overall shortest latencies in the SA group injected with salicylate after acquisition, but before extinction, relative to the groups injected before both sessions with either salicylate (SB) or saline (controls). Analysis of the pause latencies in extinction indicated the presence of main effects of drug treatment $[F(2,15)=4.5, p<.03]$ and blocks of trials $[F(5,75)=38.21, p<.0001]$, but no interaction, which is confirmed by the generally parallel curves of all groups in Figure 3. Duncan tests confirmed that the SA group had shorter latencies than the other two groups on the second block of trials, while the SA group differed from the control group, but not from the SB group on the fourth block. Accordingly, while all groups had virtually identical pause latencies by the end of acquisition training, the SA group shows shorter latencies from the very beginning of the extinction session, which is preserved to the end of the extinction session.

Figure 4 shows the mean trial latencies in extinction blocked into sixths of extinction trials. Analysis of these data did not yield a significant main effect of drug treatment, but the main effect of successive blocks of extinction trials $[F(5,75)=41.3, p<.0001]$ and the drug $\times$ blocks interaction $[F(10,75)=3.56, p<.0007]$ were both significant. Duncan tests revealed that on the first block of trials the SA group had significantly faster mean latency than did the SB and control groups, which did not differ from each other. On the last block, the control group
Acquisition

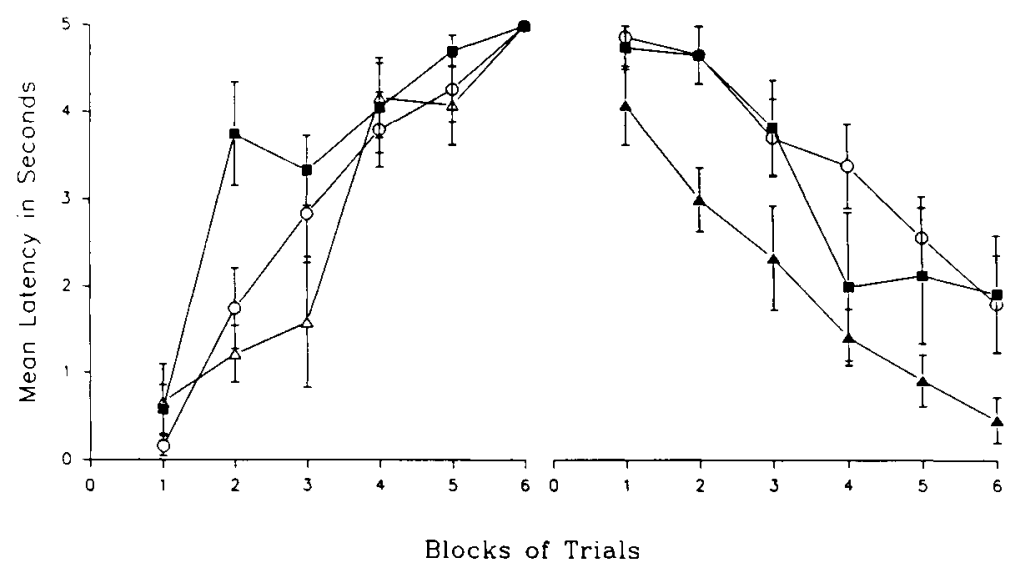

Figure 3. Mean latencies and SEMs of the pause response during successive sixths of acquisition training and extinction testing in Experiment 2 for the salicylate-injected group (SB, filed squares), the group receiving the saline vehicle prior to both sessions (controls, open circles), and the SA group injected with saline before acquisition training (open triangles) and salicylate prior to the extinction session (filled triangles). 


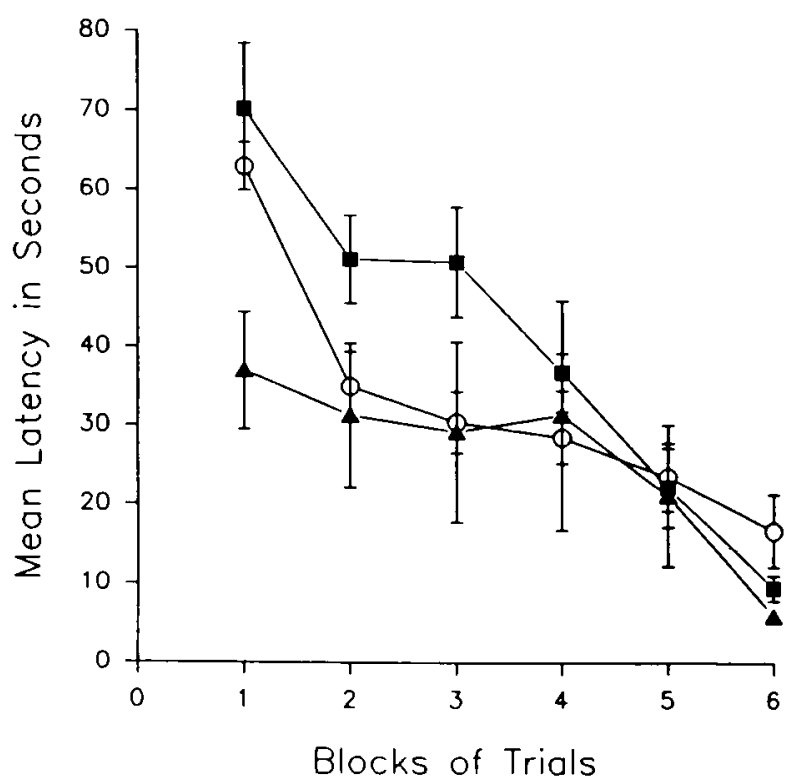

Figure 4. Mean trial latencies and SEMs during successive sixths of extinction of the suppression behavior for the two groups injected with salicylate (SB, filled squares; SA, closed triangles) and the group receiving the saline vehicle only prior to the session (controls, open circles) in Experiment 2.

differed from the SA but not the SB group, while the latter groups did not differ from each other.

The results of this experiment were quite consistent with predictions from the adult trends in the study of salicylateinduced disturbances of conditioned lick suppression (Jastreboff, Brennan, et al., 1988). During acquisition, the salicylate tended to affect the suppression. This assertion is supported by high SEMs of the acquisition trial latencies in the SB group overall and, more clearly, by the trend toward longer pause latencies during acquisition (Figure 3). Both the SA and the control groups, injected with saline before the acquisition session, were indistinguishable. In extinction, the only group that experienced a change in experimental contingencies was the SA group, which was injected with salicylate, and this group differed from the others. This finding was seen especially on the very first block of extinction trials (Figure 4) and on all blocks of the pause latency measure (Figure 3). The notion that a salicylate-induced, internal sound provided SA subjects with a novel stimulus, signaling a change from the previous session, offers a reasonable explanation of the SA behavior during extinction.

\section{GENERAL DISCUSSION}

Since Jastreboff, Brennan, et al. (1988) showed evidence of behavioral measures of drug-induced phantom auditory sensation, an animal model for the clinical presentation of tinnitus is available. Their findings offered a promising direction for the study of tinnitus and related auditory pathologies. Although a number of questions arise when one attempts to measure through objective ob- servations a phantom experience in animals, the behavioral measure of drug-induced phantom sound affords an intriguing perspective on psychological events.

Within this context, the present study extends the animal model by accommodating the dramatic growth in the auditory system and its functional role in the behavioral repertoire of the developing rat. Specifically, the present limits of age range, from 14 days until 33 days, define the time of initial auditory-mediated behavior until adultlike responses. It is not unsurprising that this period corresponds to the rapid growth of other brain centers and neurological markers, such as myelinization, which subserve other important behaviors such as memory.

Since the behavioral experiments suggest that the interval between 14 and 21 days of age may comprise a critical period of functional use of auditory stimuli, a recent study was undertaken to examine electrophysiological responses at ages corresponding to those used in the behavioral experiments (Brennan, Byrnes, \& Jastreboff, 1991; Jastreboff, Chen, Hu, \& Jastreboff, 1992). Briefly, using click stimuli with the rate of $20-22 \mathrm{~Hz}$, cochlea microphonic potentials (CM) and compound action potential (CAP) were recorded in a total of 70 rat pups at ages 13-30 days. Recordings were made before and after salicylate injections, following the analogous time relationships as in the behavioral study. Both the CAP and the CM results support the notion that the auditory system undergoes dramatic change during the age levels of present behavioral interest, and the most rapid change $o c$ curs at around 15-17 days of age, although even after 21 days, the auditory system is not fully developed.

These experiments provide support for our understanding of the ontogeny of the auditory system in two ways. First, the tasks and procedures were successful in demonstrating reliable acquisition and extinction of conditioned behavior in rats as young as 14 days of age. Moreover, this method provides an appropriate analogy to adult behaviors showing clear salicylate-induced phantom auditory experience. Second, the data suggest that the age range used in the preliminary study may represent a critical period for the emergence of salicylate-induced auditory effects.

\section{REFERENCES}

Brennan, J. F., Byrnes, J. J., \& Jastreboff, P. J. (1991, November). Salicylate induced phantom auditory effects on reinforced behavior in weanling and post-weanling rats. Paper presented at the meeting of the International Society for Developmental Psychobiology, New Orleans.

Collier, A. C., Mast, J., Meyer, D. R., Jacobs, C.-E. (1979). Approach-avoidance conflict in preweanling rats: A developmental study. Animal Learning \& Behavior, 7, 514-520.

Durrant, J. D., \& Lovrinic, J. H. (1984). Measurements of sound. In J. P. Butler (Ed.), Bases of hearing science (pp. 52-84). Baltimore: Williams \& Wilkins.

Flower, R. J., Moncada, S., \& Vane, J. R. (1980). Analgesicantipyretics and anti-inflammatory agents: Drugs employed in the treatment of gout. In A. G. Gilman, L. S. Goodman, \& A. Gilman (Eds.), The pharmacological basis of therapeutics (6th ed., pp. 682-698). New York: 'Macmillan. 
Foreman, N., \& Altaha, M. (1991). The development of exploration and spontaneous alternation in hooded rat pups: Effects of unusually early eyelid opening. Developmental Psychobiology, 24, $521-537$.

Jastreboff, P. J., \& BrenNan, J. F. (1992). Animal model of tinnitus: Recent developments. In J.-M. Aran \& R. Daum (Eds.), Proceedings of the IV International Tinnitus Seminar, Bordeaux 1991 (pp. 283292). Amsterdam: Kugler \& Ghedini.

Jastreboff, P. J., Brennan, J. F., Coleman, J. K., \&asaki, C. T. (1988). Phantom auditory sensation in rats: An animal model of tinnitus. Behavioral Neuroscience, 102, 811-822.

Jastreboff, P. J., Brennan, J. F., \& Sasaki, C. T. (1991). Quinine induced tinnitus in rats. Archives of Otolaryngology-Head \& Neck Surgery, 117, 1162-1166.

Jastreboff, P. J., Chen, G.-D., Hu, S.-M., \& Jastreboff, M. M. (1992). Effects of salicylate on the cochlear potentials during development in rats. Abstracts of 15th Midwinter Research Meeting, Association for Research in Otolaryngology, 134.

Jastreboff, P. J., Issing, W., Brennan, J. F., \& Sasaki, C. T. (1988). Pigmentation, anesthesia, behavioral factors, and salicylate uptake. Archives of Otolaryngology-Head \& Neck Surgery, 114, 186-191.

Jastreboff, P. J., Hansen, R., SASAKI, P. G., \&ASAKI, C. T. (1986). Differential uptake of salicylate in serum, cerebrospinal fluid, and perilymph. Archives of Otolaryngology-Head \& Neck Surgery, 112 , 1050-1053.

JaStreboff, P. J., \& SASAKI, C. T. (1986). Salicylate induced change in spontaneous activity of single units in the inferior colliculus of the guinea pig. Journal of the Acoustical Society of America, 80 , 1384-1391.

MaIER, S. F., \& Watkins, L. R. (1991). Conditioned and unconditioned stress-induced analgesia: Stimulus preexposure and stimulus change. Animal Learning \& Behavior, 19, 295-304.
Miller, J. S., \& Spear, N. E. (1989). Ontogenetic differences in shortterm retention of Pavlovian conditioning. Developmental Psychobiology, 22, 377-387.

Mongan, E., Kelly, P., Nies, K., Porter, W. W., \& Paulus, H. E. (1973). Tinnitus as an indication of therapeutic serum salicylate levels. Journal of the American Medical Association, 226, 142-145.

MysliveceK, J., \& HassmannovÁ, J. (1990). Early inhibitory learning in the rat: I. Learning and memory development. Developmental Psychobiology, 23, 119-128.

Phifer, C. B., Denzinger, A., \& Hall, W. G. (1991). The early presence of food-oriented appetitive behavior in developing rats. Developmental Psychobiology, 24, 453-461.

Richardson, R., Siegel, M. A., \& Campbell, B. A. (1989). Effect of maternal presence on the cardiac and behavioral responses to shock in rats as a function of age. Developmental Psychobiology, 22, $567-583$.

Rocha, J. B. T., \& VENDITE, D. (1990). Effects of undernutrition and handling during suckling on shuttle avoidance and footshock escape behavior and on plasma glucose levels of young rats. Developmental Psychobiology, 23, 157-168.

VANE, J. R. (1971). Inhibition of prostaglandin synthesis as a mechanism of action for aspirin like drugs. Nature New Biology, 231 , 232-235

Weihmuller, F. B., Collier, A. C. (1990). The role of agedependent behaviors in the retention of an approach-avoidance response in preweanling rats. Developmental Psychobiology, 23, 265-283.

(Manuscript received August 17, 1992; revision accepted for publication December 9, 1992.) 